\title{
Will there be a third COVID-19 wave? A SVEIRD model-based study of India's situation
}

\author{
D Kannan ${ }^{1}$, R Gurusriram ${ }^{1}$, R Banerjee ${ }^{1 *} \mathbb{D}$, S Bhattacharjee ${ }^{2}$ and P K Varadwaj $^{2}$ \\ ${ }^{1}$ Department of Physics and Nanotechnology, SRM Institute of Science and Technology, Kattankulathur, Tamil Nadu 603203, India \\ ${ }^{2}$ Indian Institute of Information Technology (IIIT), Allahabad, Deoghat, Jhalwa, Uttar Pradesh 211015, India
}

Received: 27 May 2021 / Accepted: 28 August 2021 / Published online: 18 September 2021

\begin{abstract}
Since the first patient was detected in India in late February 2020, the SARS-CoV-II virus is playing havoc on India. After the first wave, India is now riding the second wave. As was in the case of European countries like Italy and the UK, the second wave is more contagious and at the time of writing this paper, the per day infection is as high as 400,000 . The alarming thing is it is not uncommon that people are getting infected multiple times. On the other hand, mass vaccination has started step by step. There is also a growing danger of potential third wave is unavoidable, which can even infect kids and minors. In this situation, an estimation of the dynamics of SARS-CoV-II is necessary to combat the pandemic. We have used a modified SEIRD model that includes vaccination and repeat infection as well. We have studied India and 8 Indian states with varying SARS-CoV-II infections. We have shown that the COVID-19 wave will be repeated from time to time, but the intensity will slow down with time. In the most possible situation, our calculation shows COVID19 will remain endemic for the foreseeable future unless we can increase our vaccination rate manifold.
\end{abstract}

Keywords: COVID-19; SARS-CoV-II; Epidemics; SEIRD; Model

\section{Introduction}

COVID-19 was announced as a global pandemic by World Health Organization (WHO) [1] owing to its highly contagious and pathogenicity that has been rapidly spreading throughout the world since its first reported outbreak in China in December 2019. The "first wave" of COVID-19 slowed down by September-October 2020 as was correctly predicted by three of the current authors [2]. After going down to as low as 10,000 active patients, the "second wave" picked up by the middle of March 2021 in India. Typical of the second wave seen in European countries, this is more infectious and hence more fatal [3], as it has pushed the health infrastructure of the country to its limit. As a ray of hope, the vaccination program is going on full force throughout the country [4]. On the other hand, repeat infection of COVID-19 is not uncommon either.

As it is already noted, the second wave has pushed our health infrastructure to its limit. To overcome this situation, administrations and health departments pan India, with a

*Corresponding author, E-mail: bnrj.rudra@gmail.com
1.3 billion population and an average population density of 382 persons per $\mathrm{km}^{2}$, need a very clear and thorough idea of the challenges they are facing in this time of the pandemic. An early estimation of the width of the spread and the peak will give the front-line fighters an idea of what they need to do. This will also enable the government to make decisive planning and management of resources efficiently. Hence, a detailed analysis of real data is very much helpful to estimate the possible distribution of testing kits, oxygen, medicines, hospitals, isolation centers, etc.

The motivation of the present work is to forecast the pandemic with the help of the SVEIRD model, a wellstudied model for studying the dynamics of infectious diseases. With data of more than one year in deposition, both in government and public sources, we can nowcast and forecast the dynamics of COVID-19. We have assessed different states with different population and infection densities to show the dynamics. We have chosen 3 states with high populations density (Kerala, Tamil Nadu and Uttar Pradesh) with population density $\left(p d>500 / \mathrm{km}^{2}\right), 3$ states (Goa, Gujarat and Karnataka) with medium population density $\left(300 / \mathrm{km}^{2}<p d<500 / \mathrm{km}^{2}\right)$ and 2 states (Chhattisgarh and Madhya Pradesh) with less population 
density $\left(p d<300 / \mathrm{km}^{2}\right)$. Each state in these population density regions is further categorized by infection density (id, percentage of infected population). We have taken $i d>2 \%, 1 \%<i d<2 \%$ and $1 \%>i d$ as high, medium and low infection.

Rajesh Ranjen et al. [5] have recently characterized the parameters of COVID-19 based on total positivity rate, case fatality and effective reproductive rate. They have used the SIR model for the prediction. This together with work on testing reported by Cherian et al. [6] with a more complicated version of the SIRD model shows the necessity of more testing and a dependable source for people hospitalized or in-home isolation. In a new model-based analysis, including asymptomatic and undetected population, Agarwal et al. [7] have shown the appearance of the peak for the second wave in different states of India quite accurately. In this paper, with a simpler model and a different approach, we have analyzed the same. Moreover, the possible occurrence of the third wave is also predicted.

Overall, while the largest vaccination drive is going on in the history of human civilization [8] in the country, it is crippled by a slow vaccination rate. At the same time, the government is talking about the third wave, while the second wave is far from over. In this situation, COVID19 infection dynamics would lead us for better preparation and hopefully save more life, absence of which in the second wave lead to the current chaos and death [9].

\section{Methodology}

We have employed a modified version of the standard epidemiological model for infectious disease, i.e., SEIRD model [2] to include the effect of repeated infection and vaccination. Let us describe the effect briefly.

\subsection{Data source}

The data for the daily number of people infected, recovered and deceased from January 1 to April 30, 2021, are collected from publicly available databases like covid19india.org [10] and worldometer [11]. For the overall scenario, the Indian government's websites [12,13] are consulted. From these available data, which are in comma-separated value (csv), a few pre-processing were carried out to feed the SVEIRD-based model calculation, which is written in python3. The population density was collected from the 2011 census [14].

\subsection{SVEIRD model}

To analyze the data, we have implemented susceptible (S)vaccinated (V)-exposed (E)-infectious (I)-recovered (R)dead (D) model, a modified version of SEIRD model $[15,16]$. In the latent phase of COVID-19 infection, the infected individual is not infectious. This latent period can be incorporated into a simpler SIR model by adding the latent/exposed population (E). The infected population moves from $\mathrm{S}$ to $\mathrm{E}$ and from $\mathrm{E}$ to $\mathrm{I}$ and hopefully to $\mathrm{R}$ (Fig. 1). In this model, we have considered vaccinated people are also susceptible. This is parameterized in the absence of real data, as in Eq. (4).

The dynamical equations for the SVEIRD model are:

$$
\begin{aligned}
& \frac{d S}{d t}=-\beta S I+v R-p S \\
& \frac{d E}{d t}=\beta S I-\sigma E \\
& \frac{d R}{d t}=\gamma I-v R \\
& \frac{d V}{d t}=p S \\
& \frac{d I}{d t}=\beta S I-\gamma I-v I \\
& \frac{d D}{d t}=v I
\end{aligned}
$$

with initial conditions $S(0)>0, E(0) \geqslant 0, I(0)$ $\geqslant 0, V(0) \geqslant 0$ and $R(0) \geqslant 0$. Constants $\beta, p, \gamma, \sigma, v$ are defined in Sect. 2. The mortality rate can be calculated from Covid-India data and is related to $v$. The recovery rate is inverse of mean infectiousness period that is the average time to get cured from the onset of symptoms. The recovery rate is taken as 3,4 and 5 days, as suggested by Bi et al. [17] from the studies based on Wuhan, the inverse of which gives $\gamma$ in Eq. (1).

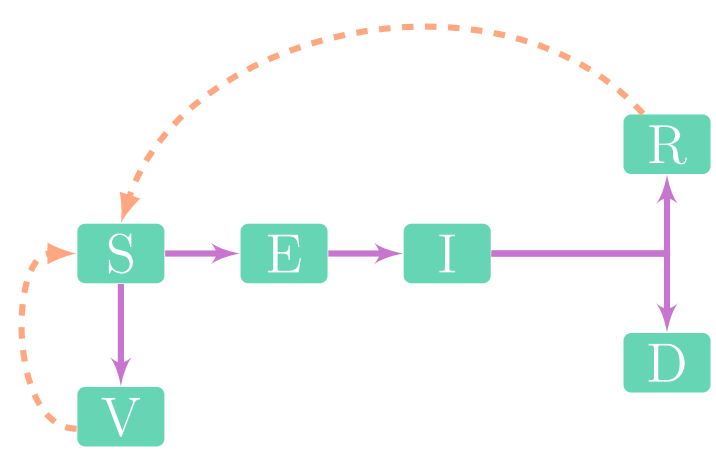

Fig. 1 Dynamics of SVEIRD model (Color figure online) 


\subsection{Descriptions of the coefficients}

Let us now describe the definitions and method of computation of the parameters for studying the population dynamics with the help of the SVEIRD model. Since the second wave has started at a different time in different states, we have taken the starting point of our calculation from January 1, 2021 (120 days).

\subsubsection{Basic reproductive ratio $\left(R_{0}\right)$}

The reproduction rate $R_{0}$ can be calculated with the daily cases starting from day 1 (January 1, 2021). Assuming that daily incidence obeys an approximate Poisson distribution, one employs the maximum likelihood estimate method. Given the observation of $\left(N_{0}, N_{1}, \ldots, N\right)$ incident cases over consecutive time units, and a generation time distribution $w, R_{0}$ is estimated by maximizing the log-likelihood

$L L\left(R_{0}\right)=\sum_{t=1}^{T} \log \left(\frac{e^{-\mu_{t}} \mu_{t}^{N_{t}}}{N_{t} !}\right)$

where $\mu_{t}=R_{0} \sum_{i=1}^{T} N_{t-i} w_{i}$. The random vector $w_{i}$ can be estimated as mean doubling time. $w$ is also called serial interval distribution [18, 19].

$R_{0}$ calculated for data up to April 30, 2021, produced a value between 1.5 and 4 [20]. The value of $R_{0}$ for India is estimated to be around 2.044 with the data up to this time limit.

\subsubsection{Serial interval $\left(T_{g}\right)$}

The serial interval is the sum of the incubation period $\left(d_{i c}\right)$ and the infectious period $\left(d_{i f}\right)$. The incubation period is the lag in days between being infected and developing the symptoms, whereas infectious period is defined as the number of days an infected person remains infectious or can infect another person, after getting infected. Doubling time is defined as time period in which the cumulative infection doubles [21, 22]. Doubling time is a function of both $T_{g}$ and $R_{0}$ [23].

$\frac{\ln (2) \times T_{g}}{R_{0}-1}$

\subsubsection{Recovery rate $(\gamma)$}

The recovery rate is defined as the inverse of infectious period $d_{i f}$. The data from WHO and other studies $[17,24-26]$ show the infectious period is between 3 and 5 days.

\subsubsection{Incubation rate $(\sigma)$}

The incubation rate is calculated by taking the inverse of mean incubation period $d_{i} n$. The data from WHO and other studies [17, 18, 25-28] show the incubation period is between 5 and 7 days.

\subsubsection{Mortality rate (v)}

The mortality rate is calculated as the number of individuals who died in a given period of time divided by the total infected in that period [29].

\subsubsection{Vaccination rate $(p)$}

The vaccination rate indicates the rate at which vaccination has occurred in each state. This is calculated as the number of people who got their second dose on or before 15 April, as immunity develops at least 2 weeks after the second dose. We have also taken vaccination efficacy of $80 \%$, as reported by the Ministry of Health and Family Welfare, Govt. of India [30]. Mathematically, this is expressed as:

$p=0.8 \times \frac{N_{v}}{N}$

where $N_{v}$ is the number of persons who got the second vaccine till April 30, 2021, and $N$ is the total population of the state.

\section{Results}

We applied the above SVEIRD model in 8 Indian states with varying populations and SARS-CoV-II infection density. Table ( $2 a$ ) gives the list of states chosen, with the population and infection density. The criteria of high, medium and low density are as described in Sect. (1). In the table, high, medium and low are color-coded as red blue and green, respectively. The same data are shown in Fig. $2 b$ as a heatmap. We have calculated the SVEIRD dynamics of each of these states. From Figs. 3, 4, 5, 6, 7, 8, 9, 10 and 11, we have shown the SVEIRD dynamics for the infected population of selected states and of India as a whole. The states with extreme cases like Maharashtra and Kerala have been omitted because, being extreme, they do not represent most states.

\subsection{States}

\subsubsection{Goa}

Is a small south Indian state and has the highest infection ratio in India $(\approx 6 \%)$ and a high population density (394 


\begin{tabular}{lll}
\hline States & $\begin{array}{l}\text { Population } \\
\text { Density }\left(/ \mathrm{km}^{2}\right)\end{array}$ & $\begin{array}{l}\text { Infection } \\
(\%)\end{array}$ \\
\hline Goa $(\S 3.1 .1)$ & 394 & 6.243 \\
Karnataka $(\S 3.1 .3)$ & 319 & 2.493 \\
Chhattisgarh $(\S 3.1 .2)$ & 189 & 2.853 \\
Tamil Nadu $(\S 3.1 .4)$ & 555 & 1.617 \\
Gujarat $(\S 3.1 .6)$ & 308 & 0.939 \\
Odisha (§3.1.5) & 269 & 1.164 \\
Uttar Pradesh $(\S 3.1 .8)$ & 828 & 0.627 \\
Madhya Pradesh $(\S 3.1 .7)$ & 236 & 0.776 \\
\hline
\end{tabular}

(a) Population density and infection/100 population of the states chosen.

Fig. 2 Choice of states and the SARS-CoV-II infection \% w.r.t. total population of the state and population density $\left(/ \mathrm{km}^{2}\right)$. In the map of $\mathbf{b}$, the red color shows the highest concentration and green the lowest. Hence, Madhya Pradesh in the bottom left corner has both low

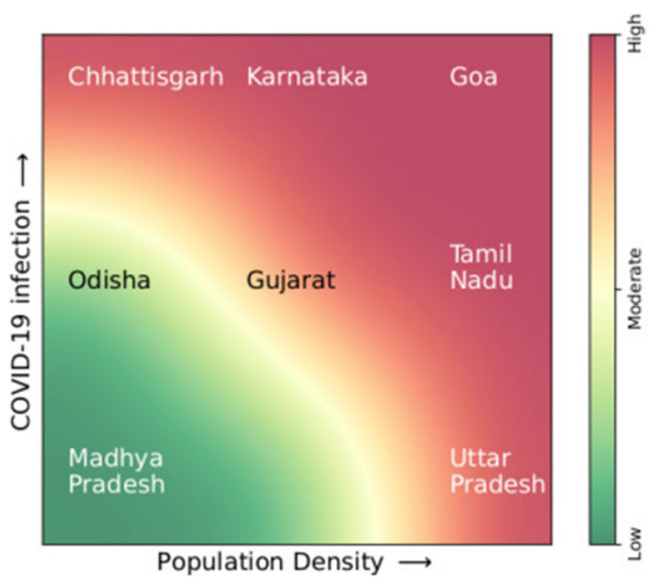

(b) Choice of states (not to the scale) as per population density and SARS-CoV-II infection (\%)

COVID-19 and population concentration. On the other hand, Goa, in the top right, has a high COVID-19 and population concentration (Color figure online)
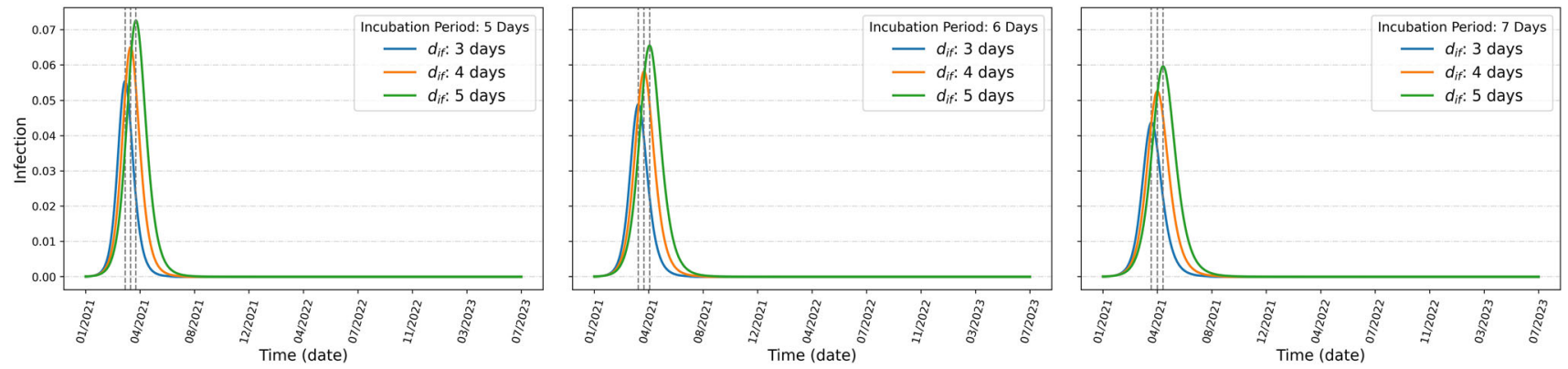

Fig. 3 SVEIRD dynamics of Goa (Color figure online)
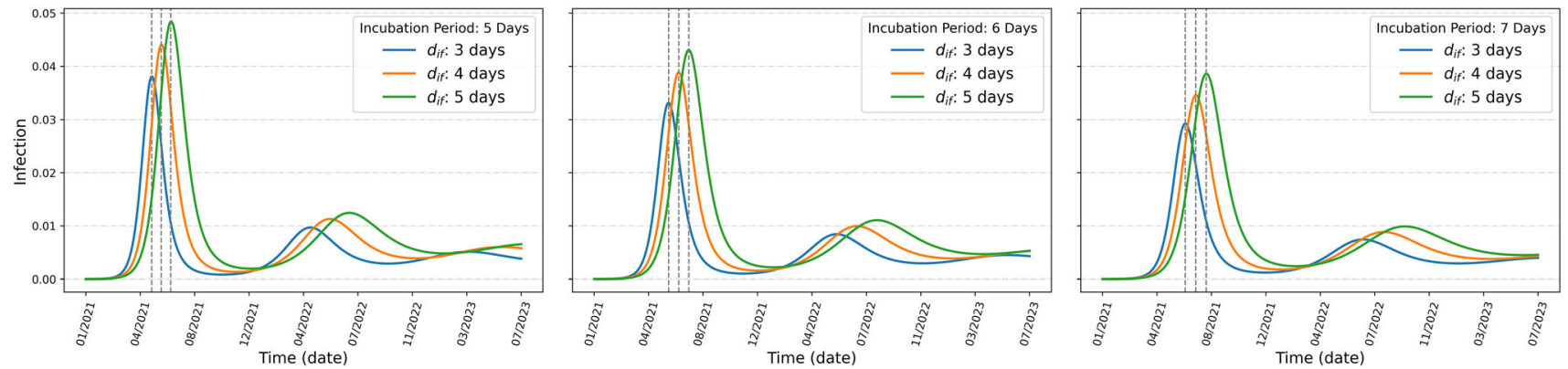

Fig. 4 SVEIRD dynamics of Chhattisgarh (Color figure online)

persons $/ \mathrm{km}^{2}$ ) as shown in Table (2a). According to our calculation, the $R_{0}$ of Goa is 2.027 . This also shows that this second wave of COVID-19 in Goa will be over by May, at the latest. The SVEIRD result for confirmed people is shown in Fig. 3. Possibly due to their small population, Goa does not show any sign of further wave in our range of study.

\subsubsection{Chhattisgarh}

Is a medium-sized state in central India, with very less population density in Indian standard. It has a population density of 189 persons $/ \mathrm{km}^{2}$ and high COVID-19 infection $(2.853 \%)$ as shown in Table (2a). 

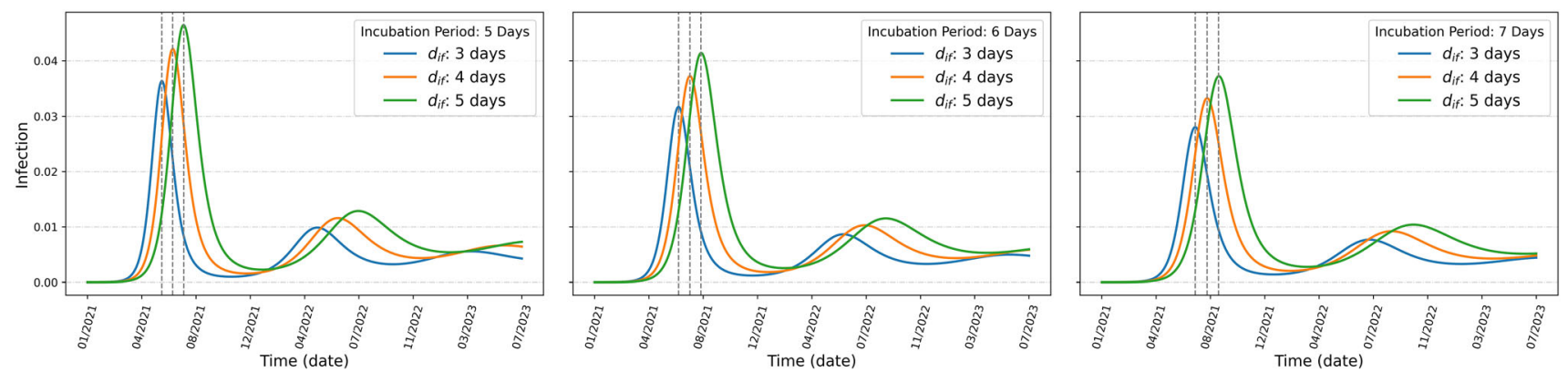

Fig. 5 SVEIRD dynamics of Karnataka (Color figure online)
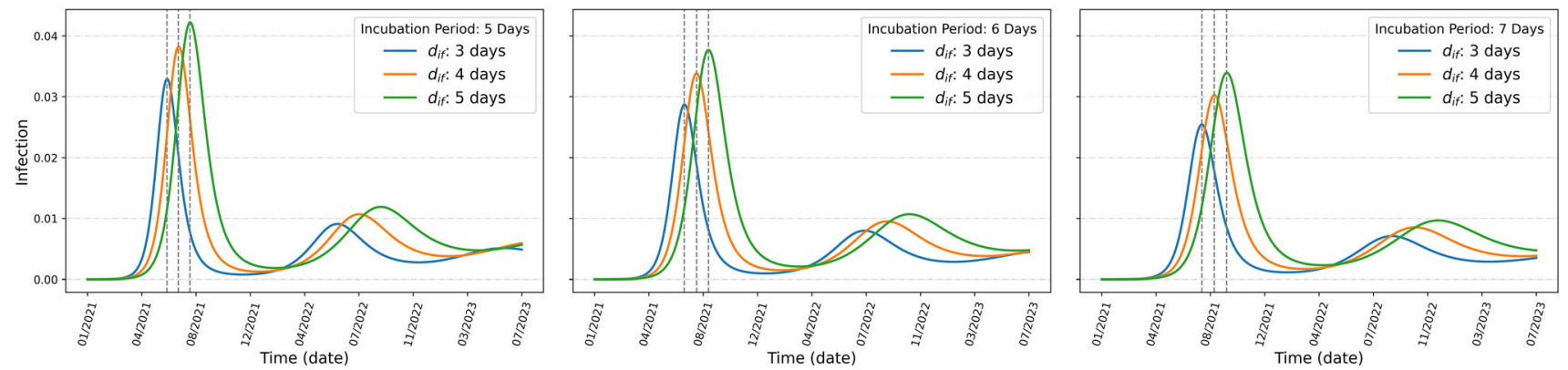

Fig. 6 SVEIRD dynamics of Tamil Nadu (Color figure online)
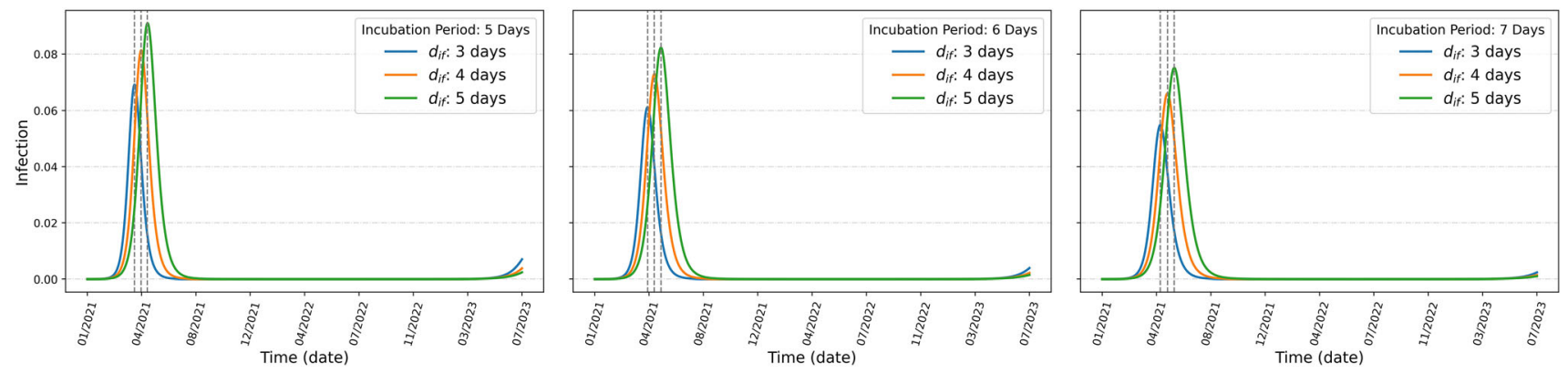

Fig. 7 SVEIRD dynamics of Odisha (Color figure online)
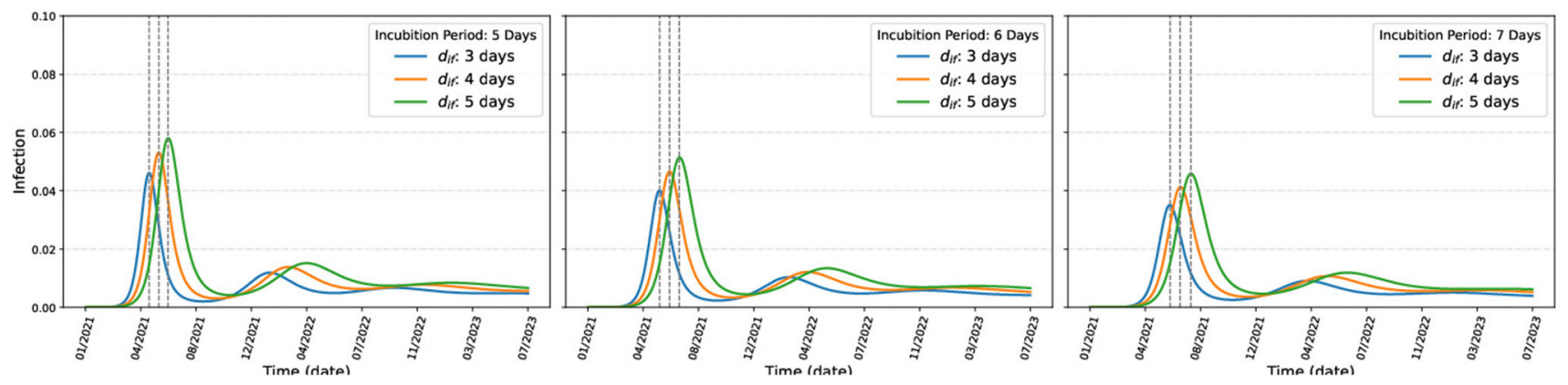

Fig. 8 SVEIRD dynamics of Gujarat (Color figure online)

As per our calculations, the $R_{0}$ of Chhattisgarh is 1.810 . Our calculation shows the peak of the second wave will arrive between the end of April and end of September, as shown in Fig. 4.
Though the most dangerous feature is that as per our calculation, the SARS-CoV-II infection may not be completely over in Chhattisgarh with the second wave, a third wave is very evident by the middle of 2022 . The data 

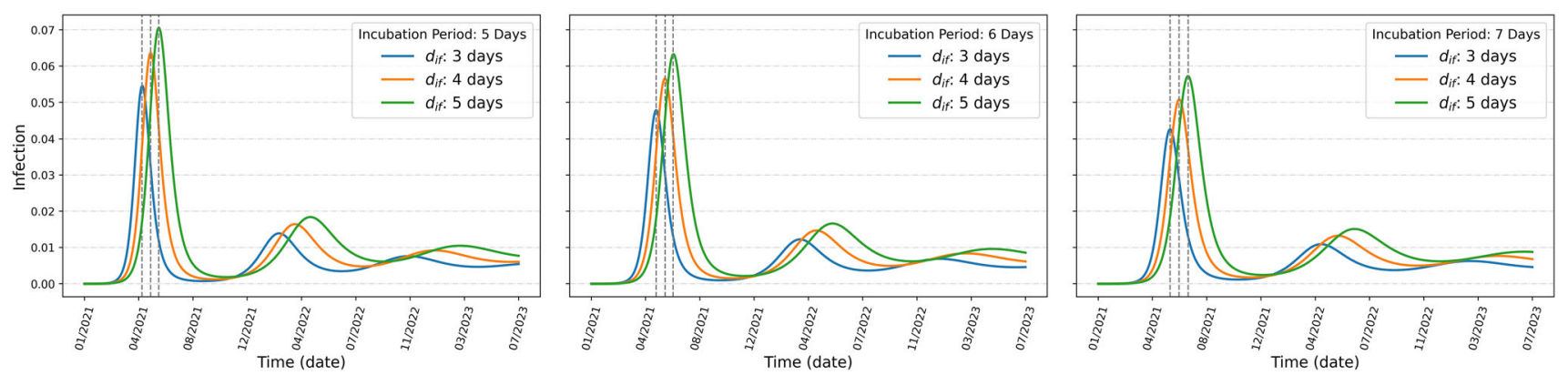

Fig. 9 SVEIRD dynamics of Madhya Pradesh (Color figure online)
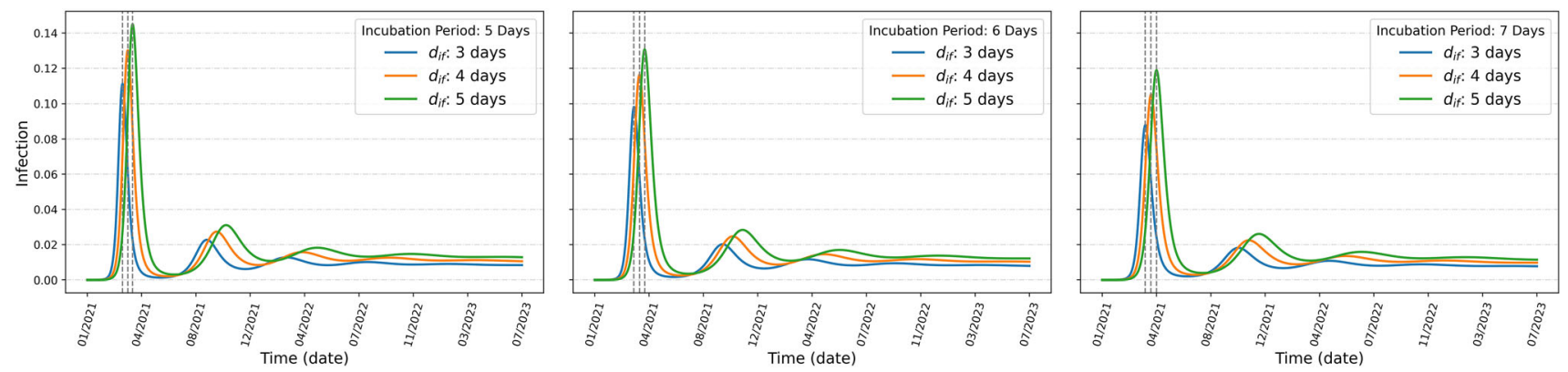

Fig. 10 SVEIRD dynamics of Uttar Pradesh (note the change of scale in y-axis) (Color figure online)
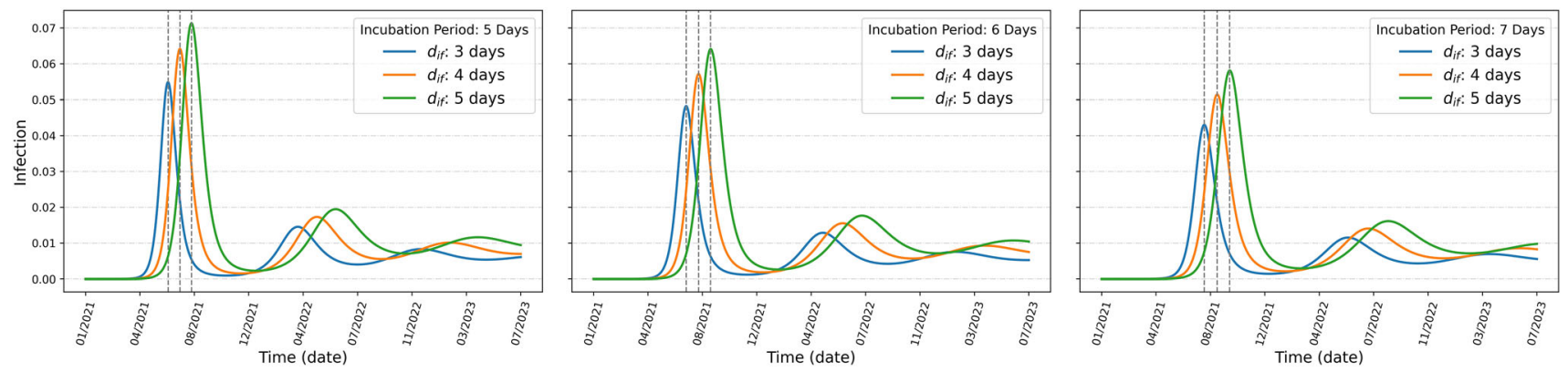

Fig. 11 SVEIRD dynamics of India (Color figure online)

actually show the COVID-19 may not be eradicated in Chhattisgarh and may live there as endemic.

\subsubsection{Karnataka}

Is one of the largest states in the Deccan peninsula, with medium (319 persons $/ \mathrm{km}^{2}$ ) but high (2.853\%) SARS-CoVII infection, as shown in Table (2a). As of April 30, 2021, Karnataka is already showing $\approx 40,000$ confirmed cases per day and there is no hint of slowing down. As per our calculations, $R_{0}$ of Karnataka is 1.769 . The peak of the second wave may happen between May and November. Just like Chhattisgarh, unfortunately, Karnataka also shows strong evidence of the third wave by the middle of next year.

\subsubsection{Tamil Nadu}

Is the southernmost state of India, with a high population density (555 persons $/ \mathrm{km}^{2}$ ) but medium infection $(1.617 \%)$, as shown in Table (2a). Tamil Nadu shows dynamics of SARS-CoV-II infection similar to its neighbor Karnataka. As per our calculations, the $R_{0}$ of Tamil Nadu is 1.705 .

The SVEIRD calculation shows the peak of the second wave may be over between May and November, similar to that of Karnataka. Also, it shows that the third wave is inevitable by the middle of next year. Unfortunately, Tamil Nadu also shows evidence that the pandemic will stay as an endemic in the foreseeable future. 


\subsubsection{Odisha}

Is a coastal state in eastern India, with a low population density (269 persons $/ \mathrm{km}^{2}$ ), as shown in Table (2a). The $R_{0}$ of Odisha is 2.245. Our study shows that Odisha has with all probability has crossed the peak of the second wave, which is between April and May, as shown in Fig. 7. Also, our calculation shows very little sign of subsequent wave in the state of Odisha.

\subsubsection{Gujarat}

Is the westernmost state of India, with a medium population density (308 persons $/ \mathrm{km}^{2}$ ) and medium SARS-CoVII infection ratio $(0.939 \%)$, as shown in Table (2a). Our calculation shows $R_{0}$ of Gujarat.

The SVEIRD-based dynamics show the peak of the second wave will appear between May and July, as shown in Fig. 8. Gujarat also shows a strong signal of the subsequent third wave and also that the COVID-19 will become endemic in Gujarat.

\subsubsection{Madhya Pradesh}

Is the second-largest state of India. It has a low (235 persons $\left./ \mathrm{km}^{2}\right)$ population density and a low $(0.776 \%)$ COVID19 infection. The $R_{0}$ of Madhya Pradesh is 2.054 , as shown in Table (2a).

Our SVEIRD calculation shows the peak of the second wave will be reached between April and September, as shown in Fig. 9. Our calculation further shows that between the second and possible third wave, Madhya Pradesh will be virtually a COVID-19-free state. The

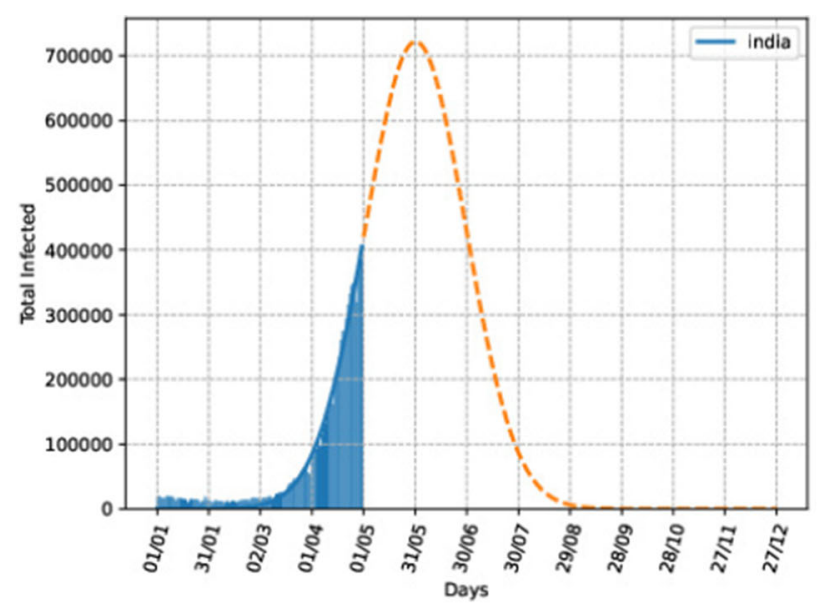

(a)

Fig. 12 (a) Gaussian fit of per day infection in India. (b) Change of SARS-CoV-II infection with the increase in vaccination rate in India. The line shows the increase in the rate of vaccination times the infection will start again by the end of December 2021 and will continue as endemic.

\subsubsection{Uttar Pradesh}

Is the Indian state with the highest population. It has a very high $\left(828\right.$ persons $\left./ \mathrm{km}^{2}\right)$ population density with very low COVID-19 (0.627\%), as shown in Table (2a). Our calculation shows the $R_{0}$ of Uttar Pradesh is 3.217 , which is the highest among the states studied.

The SVEIRD calculation shows the second wave has crossed its peak in April, as shown in Fig. 10. This matches the real data. This happy finding is overridden by the fact that Uttar Pradesh will be among the first to hit by the third wave, which may start as soon as August 2021. As common in other populous states, SARS-CoV-II will remain endemic in Uttar Pradesh as well.

In this section, we have discussed the result of the SVEIRD model, based on the data available as of April 30, 2021. Our calculation shows the higher the incubation period, the higher will be the peak, but it will also come later. Depending upon the status of the second wave and vaccination, the third wave may come within 4 to 6 months from the second wave peak. With the current spreading, our calculation shows states like Odisha and Goa will not have the third wave in near future. On the other hand, for states like Uttar Pradesh, the onset of the third wave may be as early as August 2021.

We also want to emphasize that this calculations are based on each state separately, with no human travel between them. The interstate travel may change the scenario of onset of the third wave for each state.

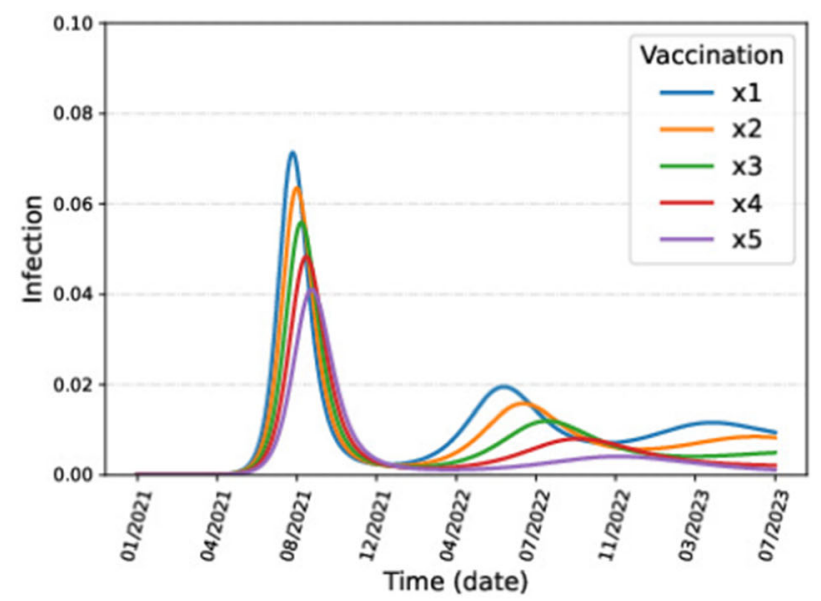

(b)

current rate. This is done for dynamics of India (Fig. 11) with an incubation period of 5 days and $d_{i f} 5$ days) (Color figure online) 


\subsection{India}

Now, let us consider the status of India as a whole. The country with the second-highest population in the world and very high population density, along with the unique socio-economic structure, it is almost impossible to maintain a social distance in India without a lockdown. This has led to a catastrophe in India, with the health system running at its limit. Our calculation shows the $R_{0}$ of India is 2.044.

The SVEIRD calculation shows India will pass the peak of the second wave between June and October. The third wave will start very soon after that, between December 2021 and April 2022, as evident from Fig. 11.

A simple Gaussian fitting with data up to April 30, 2021, shows the peak of the second wave will be around the end of May [Fig. 12(a)], with per day infection could be as high as 700,000 persons.

In the period of study, the mean doubling time is 20 days [2]. This is consistent with the prediction of Fig. 12a as the rate of infection on April 30, 2021, is around 400,000.

Finally, we have estimated the increase in the rate of vaccination we have to make to avoid the third wave. We have taken incubation period of 5 days and $d_{i f}$ of 5 days for this study. Our calculations show [Fig. 12(b)] with the increase in vaccination rate, the infection will slow down. To avoid third wave, we have to increase the vaccination more the 5 times of the current vaccination rate.

\section{Conclusions}

We have studied 8 states with varying population density and infection density pan India. Taken vaccination drive and repeat infection into consideration, we have shown the dynamics of SARS-CoV-II infection. We have shown that the third wave is inevitable and will be coming very soon. Though our dynamics shows, the third wave will infect less number of people. It may appear as early as August 2021 in few Indian states. Most of the states will see the third wave between December this year and April next year. However, we have to keep in mind that the SARS-CoV-II virus is still involving and with highly contagious nature of the virus can make the third wave come sooner and bigger. There is still no conclusive proof of the longevity of the antibody created due to vaccine or infection. Until we get everyone vaccinated, a single superspreader may create catastrophe as is in the case of the second wave. Accelerating vaccination programs can reduce and even can wipe out the possibility of a third wave. Our study shows the mask and social distancing as a normal part of our daily life for the foreseeable future.
Acknowledgements DK, GR and RB acknowledge the infrastructural supports and High Performance Computing Center, SRM Institute of Science and Technology, for providing the computational facility. SB and PV acknowledge the infrastructural supports provided by IIIT Allahabad.

\section{Declarations}

Conflict of interest We declare that we have no conflicts of interest.

\section{References}

[1] https://www.who.int/dg/speeches/detail/who-director-general-sopening-remarks-at-the-media-briefing-on-covid-19-11-march2020. Accessed on 6th May, 2021

[2] R Banerjee, S Bhattacharjee, P K Varadwaj, Quant. Biol. (2021). https://doi.org/10.15302/J-QB-021-0260.

[3] A. Borghesi, S Golemi, N Carapella, A Zigliani, D Farina, R Maroldi, Infectious Diseases 53, 370 (2021). https://doi.org/ 10.1080/23744235.2021.1884745

[4] S Bagcchi, The Lancet. Infectious Diseases 21323 (2021)

[5] R Ranjan, A Sharma, M K Verma, medRxiv (2021). https://doi.org/10.1101/2021.04.17.21255665

[6] P Cherian, S Krishna, G I Menon, medRxiv (2021). https://doi. org/10.1101/2020.12.31.20249106

[7] M Agrawal, M Kanitkar, M Vidyasagar, arXiv e-prints (2021)

[8] https://pib.gov.in/PressReleasePage.aspx?PRID=1689112. Press Release on 16/01/2021, Accessed on 12/05/2021

[9] http://hdr.undp.org/en/content/global-preparednessand-vulnerability-dashboards. Accessed on 12 May,2021

[10] https://www.covid19india.org/

[11] Worldometers.info. Total coronavirus cases in India. https://www.worldometers.info/coronavirus/country/india/ (2021). Accessed on 6th May, 2021

[12] Covid19 statewise status. https://www.mygov.in/corona-dat a/covid19-statewise-status (2020). Accessed on 6th May, 2021

[13] https://www.mohfw.gov.in/ (Accessed on 6th May, 2021)

[14] https://censusindia.gov.in/ (2011). Accessed on 6th May, 2021

[15] M Keeling, P Rohani, Modeling infectious diseases in humans and animals (Princeton: Princeton University Press, 2011)

[16] J S Weitz, J Dushoff, Scientific Reports 58751 (2015). https://doi.org/10.1038/srep08751

[17] Q Bi, et al, The Lancet Infectious Diseases 30991 (2020). https://doi.org/10.1016/S1473-3099(20)30287-5

[18] S A Lauer, et al, Annals of Internal Medicine 172577 (2020). https://doi.org/10.7326/M20-0504

[19] J Wallinga, P Teunis, American Journal of Epidemiology 160 509 (2004). https://doi.org/10.1093/aje/kwh255

[20] T Bhatnagar, et al, Indian Journal of Medical Research 151190 (2020). https://doi.org/10.4103/ijmr.ijmr_504_20

[21] E Vynnycky, R White, An Introduction to Infectious Disease Modelling (Oxford: Oxford University Press, 2010)

[22] R Anderson, M Robert, Infectious Diseases of Humans (Oxford: Oxford University Press, 1992)

[23] http://www.interfetpthailand.net/file/June2007/Presenta tion/Dr.Derek/cummings.simulation.day.1.pdf

[24] L Ferretti, et al, Science 368 eabb6936 (2020). https://doi.org/ 10.1126/science.abb6936

[25] J T Wu, K Leung, G M Leung, The Lancet 395689 (2020). https://doi.org/10.1016/S0140-6736(20)30260-9

[26] K Prem, et al, The Lancet Public Health 5 e261 (2020). https://doi.org/10.1016/S2468-2667(20)30073-6 
[27] Q Li, et al, New England Journal of Medicine 3821199 (2020). https://doi.org/10.1056/NEJMoa2001316

[28] J A Backer, D Klinkenberg, J Wallinga, Euro Surveill. 25 2000062 (2020). https://doi.org/10.2807/1560-7917.ES.20 20.25.5.2000062

[29] S Chatterjee, A Sarkar, S Chatterjee, M Karmakar, R Paul, Indian Journal of Physics (2020). https://doi.org/10.1007/ s12648-020-01766-8.
[30] https://www.mohfw.gov.in/covid_vaccination/vaccination/faqs. html\#what-to-expect-before-vaccination-2 (Accessed on 6th May, 2021)

Publisher's Note Springer Nature remains neutral with regard to jurisdictional claims in published maps and institutional affiliations. 